\title{
El modelo de valuación de activos de capital aplicado a mercados financieros emergentes \\ El caso de México 1997-2006
}

\author{
Édgar Sansores Guerrero*
}

\begin{abstract}
Resumen
Esta investigación tiene por objetivo determinar la validez, desde el punto de vista estadístico y financiero, de los supuestos básicos del modelo de valuación de activos de capital (CAPM, en inglés). También se evalúa su aplicación como modelo de formación de carteras de inversión en el mercado mexicano de valores. Los supuestos evaluados son los concernientes a la estandarización del marco que rodea la decisión, que son el criterio media-varianza, la eficiencia del mercado y las evaluaciones que realizan los inversionistas acerca de las distintas combinaciones de rendimiento esperado y riesgo de sus inversiones.
\end{abstract}

Se concluye que, aunque el CAPM ha demostrado su eficiencia como herramienta de pronóstico en economías desarrolladas, aún no se comprueba su pertinencia en mercados emergentes. En México, por ejemplo, de acuerdo con esta investigación, existen otros factores de riesgo tales como inflación, inseguridad y clima político.

Dadas las características de los mercados emergentes, el análisis con este modelo resulta inadecuado, pues distorsiona el funcionamiento del mercado de valores.

Palabras clave: mercado de valores, modelo de valuación de activos de capital (CAPM), riesgo, mercados emergentes, rendimiento.

Fecha de recepción: 24/09/2007

Fecha de aceptación: 02/06/2008

The capital assets valuation model applied to emerging financial markets.

The mexican case 1997-2006

\section{Abstract}

The purpose of this paper was to determine the validity of the basic assumptions of the Capital Asset Pricing Model (CAPM), from a statistical and financial perspective. In addition the application to the Mexican Stock Market as a model to select investment portfolios was

*Profesor-Investigador de la Universidad de Quintana Roo.

Correo electrónico: edsan@uqroo.mx

No. 226, septiembre-diciembre 2008:93-111 
Édgar Sansores Guerrero

evaluated. The following assumptions were tested: the mean-variance criteria as a standardization of the decision making framework, market efficiency, and the assessment investors follow according to investments risk and return.

It is concluded that, even though CAPM has proved to be efficient as a forecasting tool in strong economies, it still has to prove its fitness for emerging markets. According to this investigation, in Mexico other encountered risk factors are inflation, insecurity and political environment, among others.

Given the properties of emerging markets, an analysis using the CAPM model turns out to be unfit as it distorts the stock market operations.

Key words: stock market, Capital Asset Pricing Model (CAPM), risk, emerging markets, return.

\section{Introducción}

ctualmente, los analistas del mercado de valores utilizan como instrumento prinAcipal el "Modelo de Valuación de Activos de Capital". Sin embargo, dadas las características distintivas de los mercados emergentes, el análisis a través de este modelo puede resultar inadecuado y, por tanto, ser un factor que perturbe el adecuado funcionamiento del mercado de valores: dado que las expectativas de los inversionistas se forman a través de observar el mercado con este modelo, los precios de las acciones del mercado puede no ser el adecuado reflejo del riesgo y rendimiento que éstas entrañan.

El presente trabajo de investigación tiene por objetivo determinar la validez, desde el punto de vista estadístico, de los supuestos básicos del modelo concernientes a la estandarización del marco que rodea la decisión, la eficiencia del mercado y las evaluaciones que realizan los inversionistas acerca de las distintas combinaciones de rendimiento esperado y riesgo de sus inversiones.

Para el cumplimiento de este objetivo se formula la siguiente hipótesis: Los supuestos del modelo no son compatibles con el mercado financiero mexicano, es decir, el mercado no es informativamente eficiente y por tanto el modelo no es lineal, existen otros factores que afectan los rendimientos de las acciones, no existe una relación positiva entre riesgo y rendimiento.

Este trabajo está dividido en cinco secciones. En la sección II se analiza el modelo de valuación de activos de capital. En la sección III se realiza un análisis descriptivo de las características del mercado accionario mexicano. En la sección IV se realiza una prueba econométrica para determinar la validez de los supuestos. Por último, en la sección V, se exponen las conclusiones. 
El modelo de valuación de activos de capital aplicado a mercados financieros emergentes

El caso de México 1997-2006

\section{Modelo de Valuación de Activos de Capital (CAPM)}

El modelo de valuación de activos de capital o modelo de equilibrio de activos financieros, mejor conocido como CAPM, por su denominación en inglés (Capital Asset Pricing Model), fue desarrollado por Sharpe (1964) y Litner (1965). Ambos basaron sus estudios en las investigaciones realizadas por Markowitz y Tobin (1960), quienes afirmaron que todos los inversionistas seleccionan sus carteras a través del criterio media-varianza.

El objetivo del modelo es cuantificar e interpretar la relación que existe entre el riesgo y el rendimiento porque a través de esta relación lineal se puede establecer el equilibrio de los mercados financieros.

Como todo modelo económico, el CAPM basa su pertinencia en supuestos más o menos restrictivos, que le han permitido obtener conclusiones universalmente aceptadas. De acuerdo con Sharpe (1964), los supuestos básicos sobre los que está construido el CAPM son los siguientes:

a) Es un modelo estático, es decir, existe un único período en el que los activos se negocian o intercambian al principio del período y el consumo se lleva a cabo al final del mismo cuando los activos producen un pago o rendimiento.

b) Los inversionistas que actúan en el mercado son individuos aversos al riesgo que maximizan la utilidad esperada en un solo período, es decir, la función de utilidad esperada se supone biparamétrica, dependiente exclusivamente de la esperanza matemática y la varianza de las distribuciones aleatorias de probabilidad de los rendimientos de los activos financieros con riesgo. Aunque dicho supuesto puede derivarse de la función de utilidad cuadrática, debido a los importantes inconvenientes de dicha función para representar adecuadamente a un inversionista racional y averso al riesgo, se considera la consecuencia lógica de suponer que los rendimientos de los activos se distribuyen normalmente.

c) Las expectativas de todos los inversionistas sobre los rendimientos esperados, volatilidades y covarianzas entre los activos son las mismas. En otras palabras, los inversionistas son "tomadores de precios", presentando expectativas homogéneas sobre las distribuciones de rendimientos de los distintos activos financieros con riesgo, lo que permite considerar un único conjunto de oportunidades de inversión para todos los inversionistas, representado por la denominada frontera eficiente. Al igual que en el supuesto anterior, para que los únicos criterios de elección utilizados sean la media y la varianza de 
Édgar Sansores Guerrero

las distribuciones de rendimientos de los activos y de los correspondientes portafolios, se precisa suponer distribuciones normales de rendimientos.

d) Las cantidades disponibles de los distintos activos financieros con riesgo se encuentran fijadas como una variable exógena del modelo, es decir, la oferta de los activos financieros está dada; además, éstos se consideran negociables, perfectamente divisibles y no generan dividendos, sino simplemente ganancias de capital.

e) Existe la posibilidad de invertir en un activo libre de riesgo con oferta neta igual a cero y a cuyo rendimiento $\left(R_{\mathrm{F}}\right)$ se puede solicitar y otorgar una cantidad ilimitada de recursos.

f) El mercado es de competencia perfecta, es decir, ningún inversionista es lo suficientemente importante como para influir en los precios de los activos. Además, no existen fricciones en el mercado, ni costos de transacción, ni impuesto al capital.

g) El mercado financiero es informativamente eficiente, lo cual significa que el precio de mercado de la acción representa el consenso de ese mercado acerca del valor de la acción. Esto implica que los precios reflejan toda la información disponible tanto sobre la economía y el mercado bursátil como sobre la empresa particular.

Los primeros tres supuestos nos muestran la forma en la cual los individuos seleccionan sus portafolios, los dos siguientes establecen que todas las decisiones se toman en el mismo tiempo y para un mismo período, mientras que los últimos respectivamente estandarizan el marco que rodea la decisión, la eficiencia del mercado y las evaluaciones que realizan los inversionistas acerca de las distintas combinaciones de rendimiento esperado y riesgo de sus inversiones. Estos supuestos se fundamentan en los factores que integran al CAPM (Teorema de la separación, Línea del mercado de capitales y Línea del mercado de valores) y que se exponen a continuación.

\section{Teorema de la separación}

Tobin (1958) integra el término "activo libre de riesgo” en el proceso de la selección de inversión cuando identifica la necesidad que tiene el inversionista por mitigar la incertidumbre en sus rendimientos futuros para lo cual dicho inversionista acude a una opción de inversión segura. Éste selecciona especificando la proporción de su riqueza a invertir en el portafolio con riesgo $\left(W_{2}=1-W_{1}\right)$ y en el activo libre de riesgo $\left(W_{1}\right)$; dichas proporciones por invertir reflejarán su aversión al riesgo. 
El modelo de valuación de activos de capital aplicado a mercados financieros emergentes El caso de México 1997-2006

En la versión original de su investigación, Tobin (1958) considera el efectivo ${ }^{1}$ como un activo libre de riesgo con rendimientos y varianza cero, por lo que cualquier portafolio conformado por éste y un activo con riesgo tendrá como rendimiento:

$$
R=W_{2}\left(R_{2}+g\right)+W_{1}(0) \quad 0 \leq W_{2}<1 ; W_{2}=1-W_{1}
$$

en donde $g$ es una variable aleatoria que representa la expectativa de obtener rendimientos asociada a cada peso invertido en el portafolio. La expectativa de cualquier forma es igual a cero. Por lo tanto, si consideramos dichas expectativas tenemos:

$$
E(R)=v_{R}=W_{2} R_{2}
$$

La varianza del portafolio, $\sigma_{\mathrm{R}}^{2}$, puede ser descrita como:

$$
\sigma_{R}^{2}=W_{1}^{2} \sigma_{1}^{2}+W_{2}^{2} \sigma_{2}^{2}+2 \rho_{12} \sigma_{1} \sigma_{2}
$$

Donde $\rho_{12}$ es el coeficiente de correlación lineal de $R_{1 \mathrm{y}} R_{2} ; \mathrm{y} \sigma_{1 \mathrm{y}} \sigma_{2}$ son las desviaciones estándar asociadas con la varianzas de $\sigma^{2} \sigma_{2}^{2}$ de $R_{1}$ y $R_{2}$.

Simplificando términos: $\sigma_{R}^{2}=W_{2}^{2} \sigma_{2}^{2}$ y $\sigma_{R}=W_{2} \sigma_{2}$ porque $\sigma_{1}, \sigma_{2}$ y $\rho_{12=0}$ por definición del activo menos riesgoso. Desde $\mathrm{E}(\mathrm{R})$ y $\sigma_{R}{ }^{2}$ ambos dependen de $W_{2}, \sigma_{R}^{2}$ puede ser resuelta para $W_{2}$ y sustituida en la ecuación $E(R)=\frac{\sigma_{R}}{\sigma_{2}}$ Rescribiendo: $E(R)=\frac{R_{2}}{\sigma_{2}} \sigma_{R}$

Como mencionamos anteriormente, $\mathrm{E}(\mathrm{R})$ es una función lineal de la desviación estándar $\sigma_{R}$ y puede ser graficado para varios cambios en el coeficiente $R_{2} / \sigma_{2}$. El gráfico 1 ilustra como la decisión de incrementar $W_{2}$ propiciará una mayor utilidad, por un movimiento de $T_{0}$ a $T_{1} T_{1}$ es la solución óptima, representada por el punto tangente con $O C_{1}$. Tobin se refiere a $O C_{1}$, como el área de oportunidad.

${ }^{1}$ Monedas y billetes en circulación. 
Gráfica 1

Selección de inversión de un individuo averso al riesgo

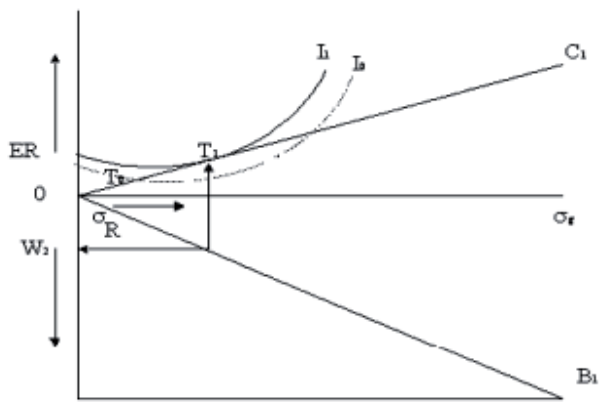

Fuente: Ayala² (1981)

Por su parte, el gráfico 1 nos muestra como los cambios en $R_{2}$ propician un cambio en la línea $O C_{1}$. Un incremento en $R_{2}$ da como resultado una nueva área de oportunidad $O C_{2}$. La nueva máxima utilidad $T_{2}$ representa un nivel mayor de riqueza esperada, $O C_{1}<O C_{2}$; para un valor especifico de $\sigma_{R}$, el inversionista añadirá proporcionalmente a su activo con riesgo por $\Delta W_{2}$, el cual es ilustrado en la figura 2 .

Gráfico 2

Selección de inversión a diferentes tasas de interés

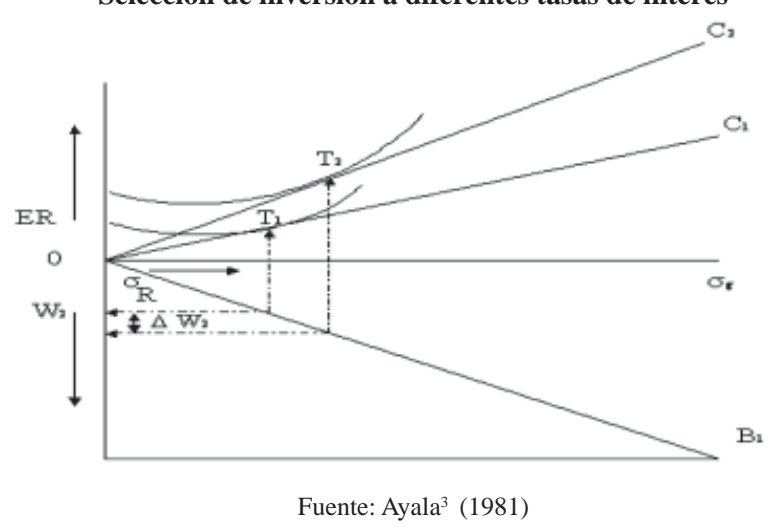

Los gráficos 1 y 2 nos muestran que un incremento en la riqueza del inversionista $\left(R_{2}\right)$ es propiciada por un incremento en la pendiente del área de oportunidad ${ }^{2}$ Mary, Ayala. “Capital Asset Pricing Model”. Universidad de Florida 1981. ${ }^{3}$ Ibid. 
El modelo de valuación de activos de capital aplicado a mercados financieros emergentes

El caso de México 1997-2006

( $\Delta W_{2}$ ). Este análisis puede ser extendido para un portafolio con múltiples activos riesgosos, el cual tendrá como rendimiento esperado $E(R)=v_{R}=W_{2} R_{2}=\sum_{i=1}^{n} W_{i} R_{2}{ }^{4}$ y varianza $\sigma_{R}^{2}=W_{2}^{2} \sigma_{R 2}^{2}{ }^{5}$.

Tobin localiza los puntos tangentes entre la isovarianza y la isomedia. Además, identifica a las combinaciones de $W_{1}$ y $W_{2}$ como el conjunto dominante, el cual se encuentra ubicado sobre la línea que va del origen hasta el área $W_{1}$ y $W_{2}$, tal y como lo muestra el gráfico 3 .

Gráfico 3

Conjunto dominante

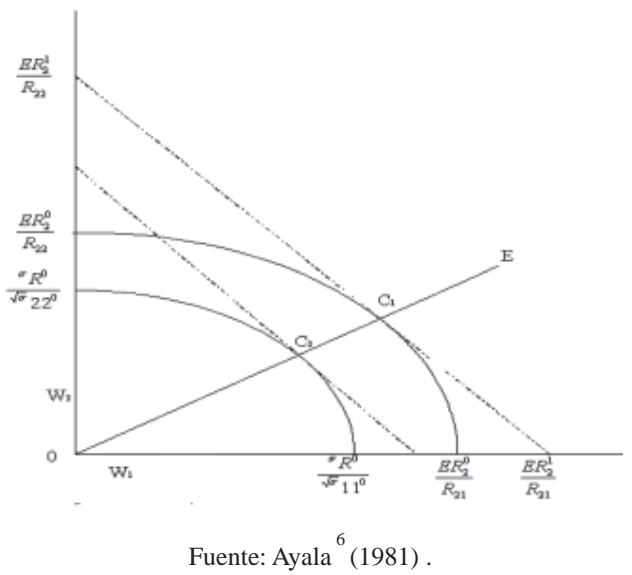

A partir de estas premisas, Tobin (1958) desarrolla el teorema de la separación, donde el conjunto dominante se ubica sobre la línea que va del origen hasta el punto E.

Bajo condiciones de igualdad se dice que el punto de E del gráfico 3 representa toda la riqueza invertida en el portafolio con riesgo. Todos los puntos localizados debajo de $\mathrm{E}$ nos muestran la distribución de la riqueza entre el portafolio riesgoso y el efectivo. El cociente de $O C^{0}$ / $O E$, por ejemplo, indica la proporción invertida en el portafolio riesgoso. Análogamente, $O C^{0}$ / $O E$ indica la proporción de la riqueza invertida en efectivo.

${ }^{4}$ En donde $W_{2}=\sum^{n} W_{i} \leq 1$

${ }^{5} \sigma_{i j}^{2}$ es igual a la varianza y covarianza respectivamente de los activos y $R_{2 i}$ y $R_{2 j}$. 
De hecho, cada punto sobre la línea $(0, \mathrm{E})$ es un punto sobre el área de oportunidad descrita en el gráfico 1 y que ha sido comprobado en el gráfico anterior.

De lo anteriormente expuesto, Tobin (1958) concluye que la combinación de la proporción invertida en activos no líquidos es independiente a su proporción en el balance de inversión. Dicha conclusión, denominada más tarde como el teorema de la separación, simplifica el proceso de selección del portafolio porque determina la combinación óptima de activos líquidos con activos financieros.

\section{Línea del mercado de capitales}

Como se mencionó anteriormente, la óptima combinación de un activo libre de riesgo y un portafolio riesgoso para un inversionista se localiza sobre la recta denominada “Frontera Eficiente Lineal”. Esta línea parte del punto denominado $R_{f \text {, el cual repre- }}$ senta el rendimiento del activo libre de riesgo, tal y como lo muestra el gráfico 4.

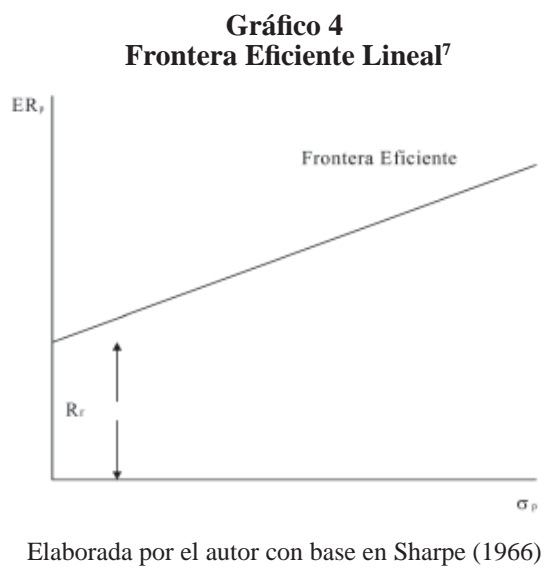

Esta línea deberá ser tangente al conjunto de portafolios eficientes del inversionista tal y como lo muestra el gráfico 5.

${ }^{7}$ Ibid. 
El modelo de valuación de activos de capital aplicado a mercados financieros emergentes El caso de México 1997-2006

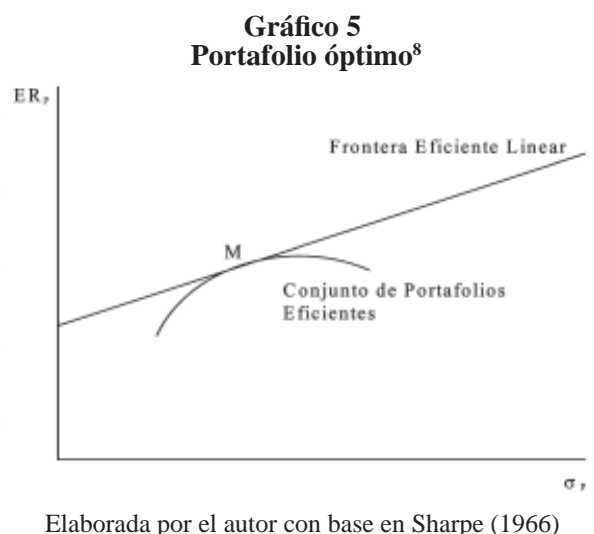

Como se muestra en el gráfico 5 el punto de tangencia con el conjunto $\mathrm{M}$ es igual al portafolio del mercado, el cual en teoría contiene una cantidad positiva de cada acción disponible. Para comprobar que $\mathrm{M}$ es el portafolio de mercado, y no cualquier otro portafolio, Sharpe (1966) determina lo siguiente. Consideremos que el punto M se sitúa sobre la frontera eficiente, por lo que los rendimientos esperados del portafolio del mercado $E\left(R_{M}\right)$ es:

$$
E R_{m}=R_{f}+\beta \sigma_{m} \quad \text { y } \quad \beta=\frac{E R_{m}-R_{f}}{\sigma_{m}}
$$

Entonces, como lo muestra Sharpe, cualquier punto sobre la línea de la frontera eficiente puede ser descrito como:

$$
E R_{p}=R_{f}+\beta \sigma_{p}
$$

Sustituyendo b: $\quad E R_{p}=R_{f}+\left(\frac{E R_{m}-R_{f}}{\sigma_{m}}\right) \sigma_{p}$, estos puntos para cualquier portafolio eficiente se ubicarán a lo largo de la línea con intercepto en $R_{f}$ y pendiente $\left(\frac{E R_{m}-R_{f}}{\sigma_{m}}\right) \sigma_{p}$. Esta pendiente es conocida como la prima de riesgo y la frontera eficiente lineal es llamada línea del mercado de capitales.

\section{Línea del mercado de valores}

Como hemos visto a través de los diferentes apartados que integran este documento, cuando un portafolio resulta eficiente su desviación estándar nos muestra el nivel de riesgo de dicho portafolio y su relación con los rendimientos esperados resulta lineal.

${ }^{8}$ Ibid. 
Cuando un portafolio es ineficiente ${ }^{9}$ esta relación lineal no se cumple. Esta situación la explicamos de la siguiente forma: Si llevamos a cabo la combinación de una acción con el portafolio de mercado, tal y como lo representa el punto AM del gráfico 6 .

\section{Portafolio resultante de la combinación del portafolio de mercado y un activo ${ }^{10}$}

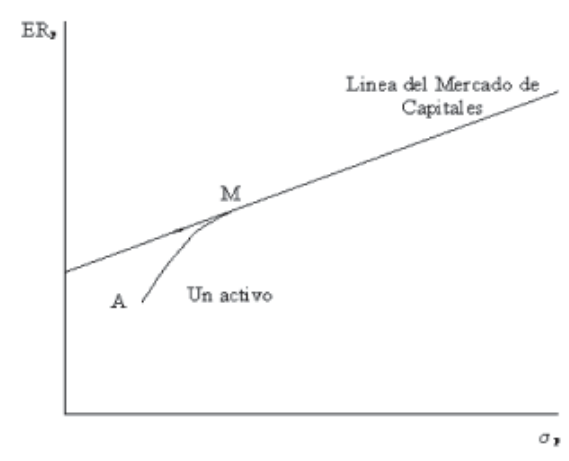

Elaborada por el autor con base en Sharpe (1966)

Sí consideramos al punto Z como cualquier portafolio ubicado sobre la línea AM, dicho portafolio tendrá como rendimiento $E R_{z}=W_{i} E R_{i}+W_{m} E R_{m} \quad$ y varianza $\sigma_{2}^{2}=W_{i}^{2} \sigma_{i}^{2}+W_{m} \sigma_{m}^{2}+2 W_{i} W_{m} \rho_{i m} \sigma_{i} \sigma_{m}$. A partir de esto podemos determinar que la pendiente de la curva $\mathrm{AM}$ al punto $\mathrm{M}$ es $\left(\frac{E R_{i}-E R_{m}}{\rho_{l m} \sigma_{i} \sigma_{m}-\sigma_{m}^{2}}\right)^{11}$. Además, en el punto AM la pendiente de la curva es la misma que la pendiente de la línea del mercado de capitales. Si esto no sucede, entonces habrá una combinación de A y M la cual será superior a la línea del mercado de capitales. Esta situación se ilustra en el gráfico 7, en donde tal combinación se encuentra representada por el punto Z*.

${ }^{9}$ Generalmente esta situación se ejemplifica a través de un portafolio integrado por un solo activo.

${ }^{10}$ Ibid.

${ }^{11}$ Para ver la formalización de esta pendiente, consúltese el anexo 2. 
El modelo de valuación de activos de capital aplicado a mercados financieros emergentes El caso de México 1997-2006

Gráfico 7

Portafolio Ineficiente ${ }^{12}$

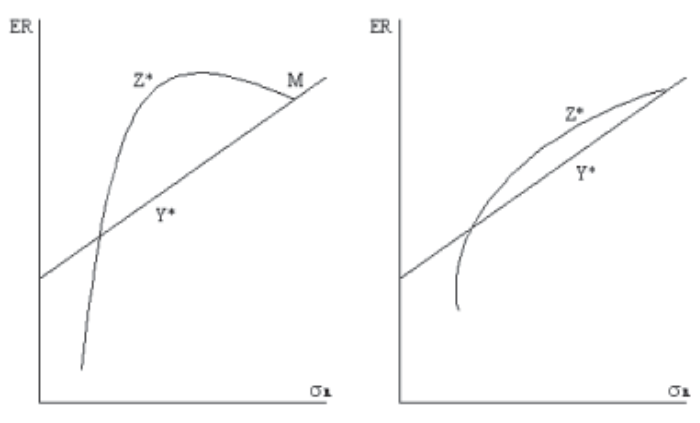

Elaborada por el autor con base en Sharpe (1966)

Para determinar la línea del mercado de valores, resulta necesario que la pendiente del punto AM sea igual a la pendiente de la línea del mercado de capitales, es decir, $\frac{E R_{m}-R_{f}}{\sigma_{m}}=\frac{\left(E R_{i}-E R_{m}\right) \sigma_{m}}{\rho_{i m} \sigma_{i} \sigma_{m}-\sigma_{m}^{2}}$. En donde $E R_{i}=R_{f}+\left(\sigma_{i m} \sigma_{i} \sigma_{m}\right)\left(E R_{m}-R_{f}\right)$, siendo $\rho_{i m} \sigma_{i} \sigma_{m}$ la covarianza de los rendimientos del activo con el rendimiento del mercado $\sigma_{i m}^{2}$; por lo cual $R_{f}+\frac{\sigma_{\mathrm{m}}^{2}\left(E R_{\mathrm{m}}-R_{f}\right)}{\sigma_{\mathrm{m}}^{2}}=E R_{i}$. Siendo la pendiente la prima de riesgo, la cual podemos establecer como $E R_{i}=R_{f}+\beta_{i}\left(E R_{\mathrm{m}}-R_{f}\right) \quad$ en donde $\beta_{\mathrm{f}}=\frac{\sigma_{\mathrm{m}}^{2}}{\sigma_{\mathrm{s}}^{2}}$, por lo que la regresión del coeficiente del CAPM resulta: $R_{i}=\alpha+\beta_{i} R_{m}+u_{i}$

$\beta_{i}{ }^{13}$ representa el riesgo sistemático del activo i, y nos muestra la sensibilidad de dicho activo con respecto a los rendimientos del mercado. El residuo $u_{i}$ es el riesgo no sistemático del activo i. El riesgo sistemático de un activo depende de su covarianza con los rendimientos del portafolio y no de su varianza.

\section{Mercado accionario en México}

El mercado accionario mexicano carece de inversionistas locales, lo que ha provocado que desde hace varios años deje de ser una alternativa de financiamiento real. Sin demanda local, depende de los grandes fondos institucionales extranjeros y los instrumentos bursátiles se caracterizan por su escasa o nula variedad. De acuerdo con

${ }^{12}$ Ibid.

${ }^{13}$ Sí resulta agresiva; Sí resulta defensiva. 
información publicada por la Bolsa Mexicana de Valores durante el período comprendido de 1990 a 2006 la inversión extranjera representó más del 45\% del valor de capitalización de la Bolsa, lo que significó el 75\% de las transacciones realizadas en dicho período. El dinero de los ahorradores estadounidenses llegó al mercado mexicano al principio de los años noventa. En ese entonces el pequeño inversionista extranjero dividía su dinero en varios fondos, unos enfocados a empresas locales, otros a emisiones de otros países y uno más con enfoque global. Después de la crisis de 1995 y otros desajustes internacionales en los años siguientes, los inversionistas aumentaron la aversión al riesgo y ahora encomiendan su dinero a fondos globales.

Por otra parte, la operatividad del mercado está concentrada en un número reducido de emisoras, ocho para ser exactos ${ }^{14}$, que en promedio intercambian acciones diariamente por un valor de cincuenta millones de pesos. Éstas representan el $80 \%$ de la actividad de la Bolsa. De las 21 casas de bolsa que operan, cinco canalizan el 52.4\% del importe operado en el mercado: GBM, Accival, Banorte, BBVA-Bancomer y Merril Lynch. En cuanto al número de empresas que están inscritas en la Bolsa ha disminuido, tal y como lo muestra el gráfico 8.

Gráfico 8

Empresas inscritas en la Bolsa Mexicana de Valores

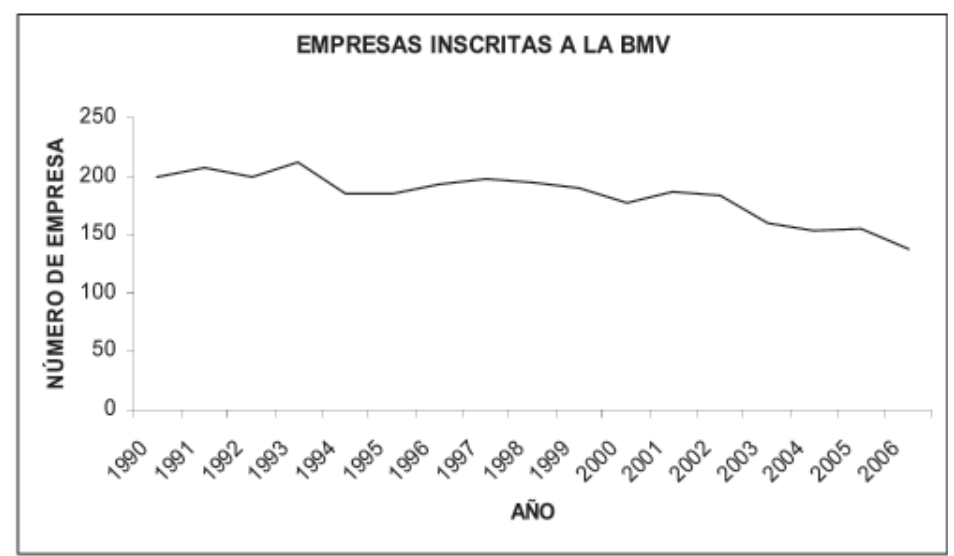

Fuente: Elaboración propia con datos de la Bolsa Mexicana de Valores.

Como se muestra en el gráfico 8, la disminución de emisoras ha originado problemas estructurales en el mercado de valores mexicano, lo que le ha impedido cumplir con su función de canalizar recursos a proyectos productivos.

${ }^{14}$ American Movil, Cemex, Banamex Accival, Telmex, BBVA-Bancomer, Wal-Mart de México, Grupo Modelo y Grupo México. 
El modelo de valuación de activos de capital aplicado a mercados financieros emergentes

El caso de México 1997-2006

\section{Evidencia empírica del CAPM en el mercado financiero mexicano}

En la actualidad, el CAPM es utilizado para la determinación de precios de acciones en la mayoría de los mercados financieros del mundo y México no podría ser la excepción; sin embargo, en la actualidad se ha suscitado un debate sobre la pertinencia de la aplicación del modelo a economías emergentes. En México existen posiciones encontradas en cuanto a su uso.

Para fines de esta investigación se evalúa la pertinencia de cada uno de los supuestos del CAPM.

\section{Metodología}

Para comprobar la pertinencia del uso del CAPM en el mercado financiero mexicano, primero analizamos el modelo propuesto por Fama y MacBeth, el cual es:

$$
\widetilde{R}_{i t}=\widetilde{\gamma}_{0 t}+\widetilde{\gamma}_{1 t} \beta_{t}+\widetilde{\gamma}_{2 t} \beta_{i}^{2}+\widetilde{\gamma}_{3 t} S_{i}+\widetilde{\varepsilon}_{i t} 2
$$

Donde:

t= Período de decisión que va de t-1 a t.

$\widetilde{R}_{i t}=$ Representa el rendimiento esperado, expresado en porcentaje, de la acción i en el período $\mathrm{t}$; esta variable es presentada en el CAPM como $E R_{i}$.

$\tilde{\gamma}_{0 t}=$ Representa el intercepto con la línea del mercado de capitales, en otras palabras, representa la tasa libre de riesgo $R_{f}$ (CETES 28).

$\widetilde{\gamma}_{1 t}=$ Representa la prima de riesgo, la cual está dada por ${ }_{i}\left(E R_{m}-R_{f}\right)$ en el CAPM.

$\beta_{i}^{2}=$ Es la variable utilizada para probar la linealidad, si el modelo es lineal entonces $E\left\{\tilde{\gamma}_{2 t}\right\}=0$

$S_{i}=$ Es la variable utilizada para probar si existe otro factor de riesgo diferente a $\beta_{i}$ y que afecta a $\widetilde{R}_{i t}$. En sentido estricto el CAPM asume que $E\left\{\widetilde{\gamma}_{3 t}\right\}=0$

$\widetilde{\varepsilon}_{i t}=$ Es el error en términos del modelo, y este asume que

$\widetilde{\varepsilon}_{i t}=0, E\left\{\varepsilon_{i t}, \varepsilon_{j i}\right\}=0 \forall_{i} \neq j, E\left\{\tilde{\varepsilon}_{i t}\right\}^{2}=\sigma_{e} \mathrm{y} \widetilde{\varepsilon}_{i t}-N\left(0, \sigma_{e}^{2}\right)$. 
Sin embargo, uno de los principales supuestos que se deben cumplir cuando se utiliza un modelo de regresión múltiple es que los errores deben ser independientes, es decir : $E\left\{\varepsilon_{i t}, \varepsilon_{j t}\right\}=0 \quad \forall_{i} \neq j$; esto no es consistente con lo planteado por Fama y MacBeth. Para solucionar este problema Fama y MacBeth agregan variables a su modelo inicial, quedando éste de la siguiente forma:

$$
\widetilde{R}_{p t}=\widetilde{\gamma}_{0 t}+\widetilde{\gamma}_{1 t} \hat{\beta}_{p}+\widetilde{\gamma}_{2 t} \hat{\beta}_{p}^{2}+\widetilde{\gamma}_{3 t} \hat{S}_{p}+\widetilde{\varepsilon}_{p t}
$$

En donde el subíndice p representa una cartera formada por varios activos. Para fines de esta prueba se formó una cartera con las acciones que conforman la muestra del Índice de Precios y Cotizaciones de la Bolsa Mexicana de Valores (IPC).

Posteriormente seguimos el modelo establecido por Black, Jensen y Scholes y Fama y MacBeth ${ }^{15}$.

Para el cálculo de $\beta_{p}$ utilizamos datos históricos del IPC y de los precios de las acciones de una cartera conformada de cuatro emisoras (TELMEX, CEMEX, WALMEX y Grupo Modelo). Para determinar $\beta_{p}$ se utilizó la siguiente regresión: $\widetilde{R}_{i t}=\alpha+\hat{\beta}_{i} \hat{R}_{m}+\widetilde{\varepsilon}_{i}$

En donde $\hat{R}_{m}$ representa el rendimiento de la cartera del mercado (IPC) y $\widetilde{R}_{i t}$ el rendimiento de nuestra cartera. Las hipótesis por comprobar fueron:

Hipótesis 1: Sí $\tilde{\gamma}_{2}=0$, el modelo es lineal.

Hipótesis 2: Sí $\widetilde{\gamma}_{3}=0$, únicamente $\beta_{i}$ afecta los rendimientos de la acción i.

Hipótesis 3: Sí $\tilde{\gamma}_{1}>0$, existe una relación positiva entre riesgo y rendimiento.

Hipótesis 4: Sí. $\widetilde{\gamma}_{0}=R_{f}$ entonces el intercepto es igual a la tasa libre de riesgo nominal en la economía mexicana (CETES 28).

De acuerdo con Black, Jensen y Scholes y Fama y MacBeth, para comprobar la segunda hipótesis, tuvimos que calcular el riesgo no sistemático de la acción i de la siguiente forma:

$$
\operatorname{Var}\left(\widetilde{R}_{i t}\right)=\hat{\beta}_{i}^{2} \operatorname{Var}\left(\widetilde{R}_{m}\right)+\operatorname{Var}\left(\widetilde{\varepsilon}_{i}\right)
$$

\footnotetext{
${ }^{15}$ Op. cit.
} 
El modelo de valuación de activos de capital aplicado a mercados financieros emergentes

El caso de México 1997-2006

En donde:

$\operatorname{Var}\left(\widetilde{\varepsilon}_{i}\right)$ representa el no- $\beta_{i}$ riesgo de la acción i. Formada la cartera, calculamos la desviación estándar de los errores $\left(S\left(\varepsilon_{i}\right)\right)$ de la cartera, la cual más tarde fue utilizada para estimar el coeficiente $\widetilde{\gamma}_{3}$. Por último, dicha hipótesis fue probada por $\hat{\beta}_{i}^{2}$.

\section{Información}

Para llevar a cabo nuestra prueba se utilizan las series históricas diarias de los precios ajustados de las acciones que conforman el IPC del 1 de octubre de 1997 al 10 de diciembre de 2006. El rendimiento del mercado está representado por la serie histórica diaria de las variaciones del IPC para el mismo período.

La serie correspondiente a la tasa libre de riesgo se integra por la tasa semanal resultado de la subasta de Certificados de la Tesorería (CETES) con vencimiento a 28 días durante el mismo período utilizado en el precio de las acciones. Es importante mencionar que durante cinco días hábiles esta tasa permanece constante, por lo que pudiera representar un problema debido a que movimientos que pudieran ser significativos en un día se ven suavizados por el uso de esta serie. Existe la alternativa de utilizar la tasa diaria ponderada de fondeo gubernamental, sin embargo, ésta es de reciente creación (1998) y podría distorsionar el análisis, por lo que no fue utilizada en esta prueba.

\section{Resultados}

Al realizar la regresión $\widetilde{R}_{i t}=\alpha+\hat{\beta}_{i} \hat{R}_{m}+\widetilde{\varepsilon}_{i}$ se obtiene este resultado:

Dependent Variable: RI

Method: Least Squares

Sample: 1114

Included observations: 114

\begin{tabular}{lrlrr}
\hline \multicolumn{1}{c}{ Variable } & Coefficient & \multicolumn{1}{c}{ Std. Error } & t-Statistic & Prob. \\
\hline \multicolumn{1}{c}{ RM } & 0.895656 & 0.501433 & 1.786191 & 0.0768 \\
\hline \hline R-squared & -0.005327 & 0.009774 & -0.545047 & 0.5868 \\
Adjusted R-squared & 0.027697 & Mean dependent var & & -0.004625 \\
S.E. of regression & 0.019016 & S.D. dependent var & & 0.105274 \\
Sum squared resid & 0.104268 & Akaike info criterion & & -1.666309 \\
Log likelihood & 1.217653 & Schwarz criterion & & -1.618305 \\
Durbin-Watson stat & 96.97959 & F-statistic & & 3.190480 \\
\hline \hline
\end{tabular}


La tabla nos muestra que la beta de nuestra cartera es de .89, por lo que se puede considerar como una inversión sensible a los movimientos del mercado, por tanto podemos esperar que el mercado refleje nuestros movimientos.

Una vez calculado el coeficiente beta procedimos a comprobar a través de la regresión $\widetilde{R}_{p t}=\widetilde{\gamma}_{0 t}+\widetilde{\gamma}_{1 t} \hat{\beta}_{p}+\widetilde{\gamma}_{2 t} \hat{\beta}_{p}^{2}+\widetilde{\gamma}_{3 t} \hat{S}_{p}+\widetilde{\varepsilon}_{p t}$ las hipótesis $\widetilde{\gamma}_{2}=0, \widetilde{\gamma}_{3}=0$, $\tilde{\gamma}_{1}>0 \quad$ y $\widetilde{\gamma}_{0}=R_{f}$

Donde:

$\tilde{\gamma}_{0}=\mathrm{C} ; \tilde{\gamma}_{2}=\mathrm{BS}, \tilde{\gamma}_{3}=\mathrm{DESV} ; \mathrm{y}=\mathrm{B} \tilde{\gamma}_{1}$

Obteniendo este resultado:

Dependent Variable: CR

Method: Least Squares

Sample: 1114

Included observations: 453

\begin{tabular}{lrlrr}
\hline \hline \multicolumn{1}{c}{ Variable } & Coefficient & Std. Error & t-Statistic & Prob. \\
\hline C & 0.125340 & 0.053840 & 2.327997 & 0.0217 \\
B & -0.346226 & 0.106860 & -3.240006 & 0.0016 \\
DS & 0.259537 & 0.067022 & 3.872419 & 0.5200 \\
\hline DESV & -3.440008 & 1.933050 & -1.779575 & 0.0077 \\
\hline R-squared & 0.189992 & Mean dependent var & & -0.001916 \\
S.Ejusted R-squared & 0.167901 & S.D. dependent var & & 0.055109 \\
Sum squared resid & 0.050270 & Akaike info criterion & & -3.108348 \\
Log likelihood & 0.277981 & Schwarz criterion & & -3.012341 \\
Durbin-Watson stat & 181.1758 & F-statistic & & 8.600359 \\
\hline \hline
\end{tabular}

El resultado muestra que para la cartera construida el coeficiente $\hat{\beta}_{i}{ }^{2}$ no resulta estadísticamente diferente a cero. Por tanto no podemos rechazar la hipótesis nula de que $H o=\widetilde{\gamma}_{2}=0$, es decir, el modelo puede ser lineal.

Como mencionamos anteriormente, la inclusión de la desviación estándar en el modelo nos permite estudiar si existen otros factores, diferentes de $\beta_{i}$, que afectan los rendimientos. En la cartera estudiada, $\widetilde{\gamma}_{3}$ fue altamente significativa así que se rechaza la hipótesis nula que $H o=\widetilde{\gamma}_{3}=0$. Lo cual confirma la existencia de otros factores que afectan a $\beta_{i}$ en el mercado mexicano. 
El modelo de valuación de activos de capital aplicado a mercados financieros emergentes

El caso de México 1997-2006

Llama la atención que el signo del coeficiente $\widetilde{\gamma}_{1}$ sea negativo, esto ha sido observado en otros mercados por otros investigadores ${ }^{16}$. Esto nos lleva a la conclusión de que el premio al riesgo de la cartera es menor al que teóricamente deberíamos observar, por lo que se rechaza la hipótesis de que existe una relación positiva entre el riesgo y el rendimiento, o lo que es lo mismo $\tilde{\gamma}_{1}>0$. Finalmente, la última hipótesis también se rechaza debido a que $\tilde{\gamma}_{0} \neq R_{f}$.

De los resultados obtenidos tentativamente se pudieron confirmar dos hipótesis:

1) la linealidad del modelo y 2) la existencia de otros factores, diferentes a $\beta_{i}$, que afectan a los rendimientos esperados.

\section{Conclusiones}

Aunque el CAPM ha demostrado su eficiencia como una herramienta de pronóstico en economías fuertes, aún no se ha comprobado su pertinencia en mercados emergentes. Sin embargo, dada las características distintivas de los mercados emergentes ${ }^{17}$, el análisis a través de este modelo resulta inadecuado y, por tanto, distorsiona el funcionamiento del mercado de valores.

Para el caso de México, de acuerdo con los resultados obtenidos en esta investigación, existen otros factores de riesgo que afectan los rendimientos de las acciones tales como inflación, inseguridad, clima político, entre otros. Además, la prima de riesgo que paga el mercado es relativamente baja con respecto a la calculada a través del modelo; es decir, existe un desequilibrio en el mercado ya que se tienen acciones sobrevaluadas y subvaluadas. Por otra parte, el nivel de la tasa de descuento de los Certificados de la Federación dista mucho de la tasa de interés libre de riesgo, en otras palabras, los CETES no representan lo que Sharpe denomina tasa libre de riesgo.

\section{Bibliografía}

Asociación Mexicana De Intermediarios Bursátiles, Operaciones bursátiles en el mercado de valores, Editado por la Asociación Mexicana de Intermediarios Bursátiles. 2001.

BLACK, F., "Capital Market Equilibrium with Restricted Borrowing”, Journal of Bussines, Núm. 10, Vol. 20, pp. 444-455, 1993.

\footnotetext{
${ }^{16}$ Jensen

${ }^{17}$ Los mercados emergentes, que por lo común se conocen en la comunidad bursátil, son los incluidos en el banco de inversión estadounidense Morgan para los mercados emergentes mundiales (MSCI GEM)
} 
Édgar Sansores Guerrero

Black, F., “Beta and Return”, Journal of Portfolio Management, Vol, 20, Núm. 10, 1993, pp. 215-230.

Black, J. Y M. Scholes, “The Capital Asset Pricing Model: Some Empirical Test. Studies in the Theory of Capital Markets”, 1972, pp. 79-124.

Bollerslev, K. "Financial Market Efficiency Test”, NBER working paper Núm. 4108, 1992.

Bollerslev, T., “Generalized Autoregressive Conditional Heteroscedasticity”, Journal of Econometrics, Núm. 31, 1993, pp. 307-327.

Burton, J., "Revisiting The Capital Asset Pricing Model”, Dow Jones Asset Management. mayo/junio, 1998, pp. 456-470.

CampBell, J., The econometrics of financial markets, Mc. Graw Hill, 2001.

Ross, S., "Economic Forces and the Stock Market", Journal of Business, Vol. 59, No. 3, pp. 383-403.

DAVID L. Y R. Russell, “Estimating Time Varying Risk Premia in the Term Structure, The arch-m Model”, Econometrica 55, marzo, 1987, pp. 391-407.

Douglas, G., "Risk in Equity Markets: An Empirical Appraiser of Market Efficiency”, Yale Economic Essays, Vol. 9. 1969, pp. 3-45.

Engle, Robert F., “Autoregressive Conditional Heteroscedasticity with Estimates of the Variance of United Kingdom Inflation”, Econometrica 55, julio, 1982, pp. 987-1007.

Feldstein, M., "Mean Variance Analysis in the Theory of Liquidity Preference and Portfolio Selection”, Review of Economic Studies, Enero de 1969, pp. 5-12.

GonZÁLEz, M., "CAPM Performance in the Caracas Stock Exchange from 1992 to 1998”, International Review of Financial Analysis, Núm. 10, pp. 334-341.

LitNer, J., “Security Prices, Risk and Maximal Gains from Diversification”, Journal Of Finance, diciembre, 1965, pp. 128-143.

MalKiel, M.,“Efficient Market Hypothesis”, citado en José Marín, Economía Financiera. Edit. Andoni Bosh.

Markowitz, H., “Portfolio Selection”, Journal of Finance, marzo, 1962, pp. 77-91.

Radcliffe, R., Investment Concepts, Analysis, Strategy, Harper \& Collins, Estados Unidos 2000. 
El modelo de valuación de activos de capital aplicado a mercados financieros emergentes

El caso de México 1997-2006

KolB, R., Inversiones, Blackwell Publishers, México 2000.

Rubinstein, M., "Securities Market Eficiency in Arrow-Debreu Economy”, American Economic Review núm. 65, pp. 812-824.

Sharpe, W., Portfolio Theory and Capital Markets, Mc Graw-Hill, 2000.

"Capital Asset Prices: A Theory of Market Equilibrium Under Conditions of Risk”, Jounal of Finance, Núm. 15, 1966, pp. 105-130.

Tobin, James, "Liquidy Preference as Behavior Towards Risk", The Review of Economic Studies, febrero, 1958, pp. 65-86.

Villegas E. Y R. Ortega, El Sistema Financiero de México, Mc. Graw Hill. México, 2002.

Weston, F. Y E. Copeland, Finanzas en Administración, Mc Graw-Hill, México, 2000.

WiLliam, J.B., The Theory of Investment Value, Harvard University Press, 1938 
